\title{
Molecular Sensitization Profile According to Proton Pump Inhibitor Response in Patients With Esophageal Eosinophilia
}

Lluncor $\mathrm{M}^{1}$, Pedrosa $\mathrm{M}^{1}$, Cancelliere $\mathrm{N}^{1}$, Rivero-Paparoni $\mathrm{D}^{1}$, Burgos A ${ }^{2}$, Fiandor A ${ }^{1}$, Pagola $\mathrm{MJ}^{1}$, Quirce $\mathrm{S}^{1}$, Caballero $\mathrm{T}^{1}$ ${ }^{1}$ Allergy Department, Hospital La Paz Institute for Health Research (IdiPaz), Madrid, Spain

${ }^{2}$ Gastroenterology Department, Hospital Universitario La Paz, Madrid, Spain

J Investig Allergol Clin Immunol 2018; Vol. 28(5): 354-358 doi: 10.18176/jiaci.0289

Key words: Eosinophilic esophagitis. Component-resolved diagnosis. Sensitization profile. Cross-sensitization.

Palabras clave: Esofagitis eosinofílica. Diagnóstico por componentes. Perfil de sensibilización. Reactividad cruzada.

Proton pump inhibitor-responsive esophageal eosinophilia (PPI-REE) and eosinophilic esophagitis (EoE) are clinically undistinguishable entities [1-3]. Component-resolved diagnosis enables simultaneous assessment of specific $\operatorname{IgE}$ antibodies to various allergens and could provide us with more accurate information about sensitization profiles in these patients [4-6]. The aim of this study was to compare the molecular sensitization profiles of patients diagnosed with EoE and PPI-REE.

We performed a retrospective cross-sectional study. The study population comprised patients followed up at the Allergy Department of Hospital Universitario La Paz, Madrid, Spain with EoE and specific IgE measured by immuno-solid-phase allergen chip (ISAC, Thermo Fisher Scientific).

The patients were recruited between January 2000 and December 2014. EoE and PPI-REE were confirmed according to guidelines [1]. Patients in whom eosinophils were not limited to the esophagus were excluded. The study protocol was reviewed and approved by Hospital La Paz Research Ethics Committee (PI-2012).

We retrospectively collected demographic variables (sex, age), data on atopic background (allergic rhinitis and/or conjunctivitis, asthma, food allergy, and atopic dermatitis), medical data (celiac disease, Helicobacter pylori infection), and clinical characteristics. Skin prick tests (SPTs) were performed with a specially designed panel for EoE, which included food extracts, aeroallergens, Anisakis simplex, and latex. A wheal diameter $\geq 3 \mathrm{~mm}$ larger than the negative control was considered positive. Serum total IgE, tryptase, eosinophil cationic protein, and peripheral eosinophils were measured. The statistical analysis was performed with IBM SPSS statistics for iOS, Version 20.0 (IBM Corp).

Sixty-one out of 82 patients with EoE who had undergone component-resolved diagnosis completed the PPI trial. PPI-REE was confirmed in $42.6 \%$ of patients and EoE in $57.4 \%$. EoE and PPI-REE patients had similar demographic 


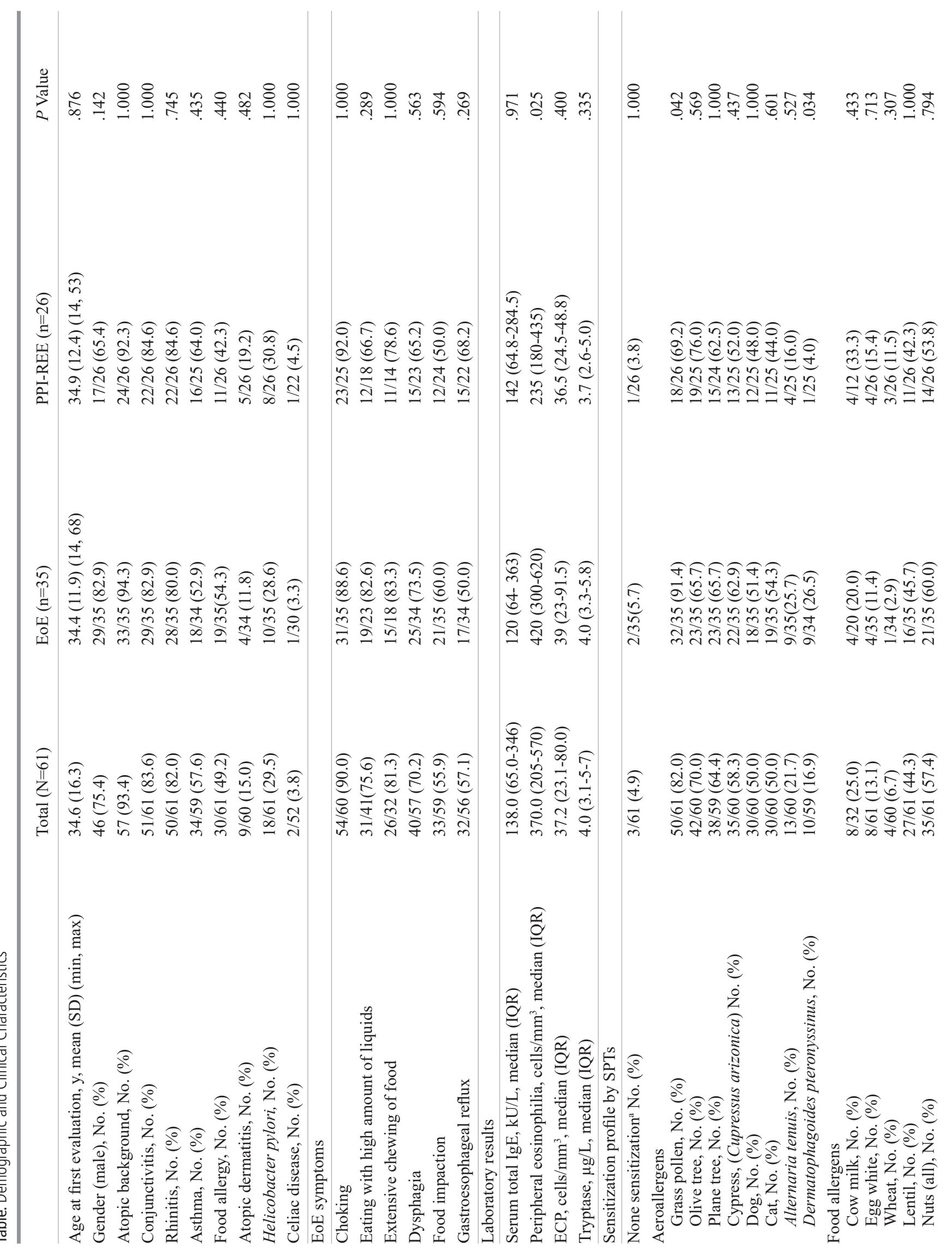




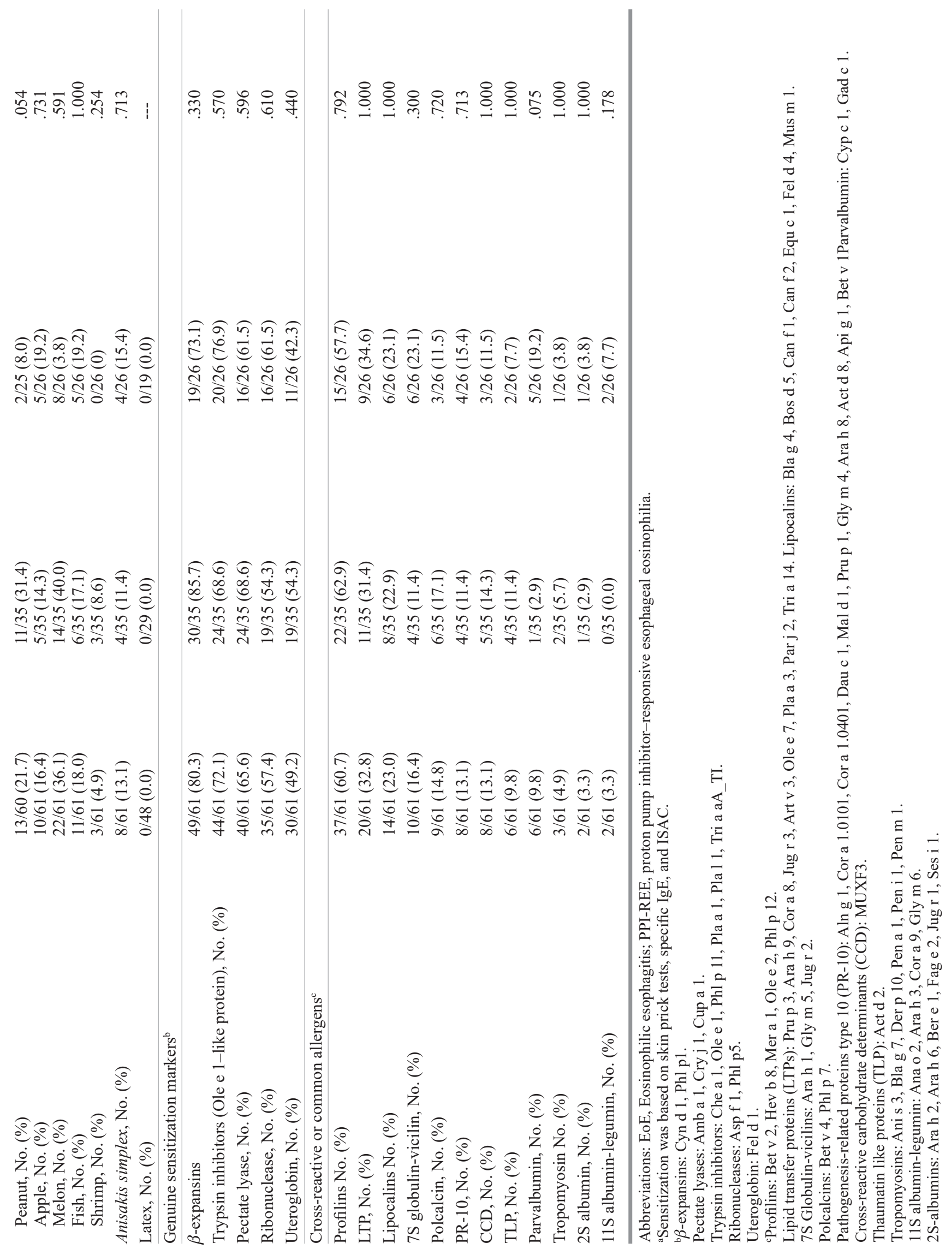


and clinical characteristics (Table), except for peripheral eosinophilia and sensitization to Dermatophagoides pteronyssinus and grass pollen, as measured by SPT. A high prevalence of sensitization was identified by SPT and/or specific IgE to aeroallergens and food allergens in both EoE patients (80.0\%) and PPI-REE patients (76.9\%).

Specific IgE antibodies against 103 and 112 allergen components were measured by ISAC in 36 and 46 patients, respectively. The prevalence of the various sensitizations was similar in EoE and PPI-REE patients (Table).

Comparison of specific IgE levels between EoE and PPIREE revealed no significant differences between the groups, except for sensitization to Can $\mathrm{f} 5$, for which higher IgE levels were observed in PPI-REE than in EoE (mean, 2.14 [3.3] ISU vs 0.44 [1.42] ISU, $P=.018$ ). Nevertheless, there were no differences in the frequency of sensitization to Can $\mathrm{f} 5$ between the groups.

We compared the molecular sensitization profile of EoE and PPI-REE patients and found that sensitization profiles and demographic and clinical characteristics were similar, as previously reported [1-3].

PPI-REE patients had lower blood eosinophilia than EoE patients (Table), although the median eosinophil count was within the normal range in both groups, as already described [1-3].

Sensitization (as determined) by SPT, to D pteronyssinus, peanut, and grass pollen was more frequent in EoE than PPIREE, although there were no differences regarding molecular sensitization. The only study to analyze IgE-ISAC in EoE and PPI-REE patients showed similar sensitization profiles in EoE and PPI-REE, although the authors did not provide details of the profiles [2].

As for markers of primary aeroallergen sensitization, we found a high frequency of sensitization to $\beta$-expansins (mainly Phl p 1), followed by trypsin inhibitors (predominantly Ole e 1) and pectate lyases (Cup a 1). Other studies also found that $\beta$-expansins in grass pollen were among the most prevalent allergens recognized by EoE patients [4-6]. The high prevalence of $\beta$-expansin, Ole e 1-like protein, and pectate lyase sensitizations in our sample may be related to the high frequency of sensitization to grass, olive, and Cupressaceae pollens in the Spanish population [7].

In relation to cross-sensitization allergens, we found a high frequency of recognition of $\operatorname{IgE}$ to profilin $(60.7 \%)$, followed by lipid transfer protein $(32.8 \%)$ and lipocalin $(23.0 \%)$. The reported frequency of sensitization to profilin is variable $(11-40 \%)$ [4-6]. The high frequency of profilin sensitization in our series, together with the high grass pollen counts in Madrid [7], may be related to the fact that the most prevalent type of pollen sensitization is to grass pollen [8]. It is not clear whether aeroallergens can produce EoE, although one study reported that EoE symptoms triggered by plant-derived foods were most frequently associated with profilin sensitization [4], whereas there is only 1 case report suggesting that profilin could be a relevant allergen in EoE [9].

In contrast to our results, PR-10 proteins have been reported to be the main cross-reactive allergens (37\%-39\%) in areas where sensitization to plant foods is related to crossreactivity with Bet $\mathrm{v} 1[4,5]$. The prevalence of sensitization to LTP was higher (32.8\%) than in other studies (2.6-11\%) [4,5] and was confirmed in another Spanish study, which showed a high prevalence of sensitization to Pru p 3 (19.4\%) [6].

This specific sensitization pattern to markers of genuine sensitization and cross-reactive allergens can be explained by differences in aeroallergen and food exposure according to the geographical area. This finding could be evaluated by comparing this population with a control group of patients with other allergic diseases.

Our study is limited by its retrospective nature and the fact that some data are missing. Nevertheless, all available data were considered for the analysis. ISAC was not systematically performed in all of the patients and it seems likely that most of the patients were sensitized to multiple allergens. Despite these limitations, our results are comparable with those of previous studies.

The strengths of the study are the large sample size in which ISAC was performed and the fact that it was the first to compare detailed sensitization profiles between EoE and PPI-REE patients.

In summary, EoE and PPI-REE patients share comparable sensitization profiles with only slight differences between them. A high frequency of sensitization to specific grass pollen was found. However, the clinical relevance of these results should be further assessed through targeted elimination diets.

\section{Funding}

The authors declare that no funding was received for the present study.

\section{Conflicts of Interest}

The authors declare that they have no conflicts of interest.

\section{References}

1. Lucendo AJ, Molina-Infante J,Arias Á, von Arnim U, Bredenoord AJ, Bussmann C, et al. Guidelines on eosinophilic esophagitis: evidence-based statements and recommendations for diagnosis and management in children and adults. United Eur Gastroenterol J. 2017;5(3):335-58.

2. Warners MJ, van Rhijn BD, Curvers WL, Smout AJ, Bredenoord AJ. PPI-responsive esophageal eosinophilia cannot be distinguished from eosinophilic esophagitis by endoscopic signs. Eur J Gastroenterol Hepatol. 2015;27(5):506-11.

3. Dellon ES, Speck O, Woodward K, Gebhart JH, Madanick RD, Levinson $S$, et al. Clinical and endoscopic characteristics do not reliably differentiate PPI-responsive esophageal eosinophilia and eosinophilic esophagitis in patients undergoing upper endoscopy: a prospective cohort study. Am J Gastroenterol. 2013;108(12):1854-60.

4. Simon D, Straumann A, Dahinden C, Simon HU. Frequent sensitization to Candida albicans and profilins in adult eosinophilic esophagitis. Allergy. 2013;68(7):945-8.

5. van Rhijn $B D$, van Ree $R$, Versteeg $S A$, Vlieg-Boerstra $B J$, Sprikkelman AB, Terreehorst I, et al. Birch pollen sensitization with cross-reactivity to food allergens predominates in adults with eosinophilic esophagitis. Allergy. 2013;68(11):147581. 
6. Armentia A, Martín S, Barrio J, Martín B, García JC, Vega JM, et al. Value of microarray allergen assay in the management of eosinophilic oesophagitis. Allergol Immunopathol (Madr); 2015;43(1):73-80.

7. Belver MT, Caballero MT, Contreras J, Cabañas R, Sierra E, Madero $R$, et al. Associations among pollen sensitizations from different botanical species in patients living in the northern area of Madrid. J Investig Allergol Clin Immunol. 2007;17(3):157-9.

8. Rodríguez del Río P, Díaz-Perales A, Sánchez-García S, Escudero C, Ibáñez M, Méndez-Brea P, et al. Profilin, a change in the paradigm. J Investig Allergol Clin Immunol. 2017;28(1):1-12.

9. Domínguez Ortega J, Pérez-Bedmar J, Rodríguez Jiménez B, Butrón M, Kindelan C, Ledesma A. Eosinophilic esophagitis due to profilin allergy. J Invest Allergol Clin Immunol. 2008;19(4):321-39.

Manuscript received October 1, 2017; accepted for publication on June 25, 2018.

Marina Lluncor-Salazar Allergy Department Hospital Universitario La Paz Paseo de la Castellana, 261 28046 Madrid, Spain

E-mail: mar_ls@hotmail.com 